\section{Atropine-resistant bradycardia due to hyperkalaemia}

\author{
T J Slade, ${ }^{1}$ J Grover, ${ }^{2}$ J Benger ${ }^{1}$
}

Symptomatic sinus bradycardia is routinely treated in the emergency department with atropine and pacing. Two cases are presented that illustrate the importance of considering hyperkalaemia, particularly in the presence of atropine-resistant symptomatic bradycardia. The administration of calcium in such cases acts to stabilise the myocardium and resolve the bradycardia. Blood gas analysis provides a rapid estimate of serum potassium concentrations, facilitating timely treatment.

\section{CASE 1}

A 68-year-old man was brought to the emergency department (ED) having collapsed after dinner. The ambulance crew recorded an initial pulse rate of 33 and blood pressure of $80 / 30 \mathrm{~mm} \mathrm{Hg}$. He received $1.5 \mathrm{mg}$ atropine pre-hospital with no discernable effect. His past medical history included hepatic failure and myocardial infarction and current medications were metoprolol, valsartan, spironolactone (dose recently increased), clopidogrel, aspirin, atorvastatin and omeprazole.

On arrival in the ED he was conscious, with a heart rate varying between 38 and 45. His arrival ECG revealed a sinus rhythm with an increased PR interval (210 ms), broadened ORS complexes and a QTc of $444 \mathrm{~ms}$ (fig 1). A venous blood gas gave a potassium result of $8.1 \mathrm{mmol} / 1$, with hyperkalaemia confirmed by a subsequent laboratory report of $8.4 \mathrm{mmol} / 1$.

A further $1 \mathrm{mg}$ intravenous atropine was given without effect. However, $5 \mathrm{mg}$ nebulised salbutamol and $2 \mathrm{~g}$ intravenous calcium chloride administered over 5 minutes caused the heart rate to increase to 68 with a blood pressure of $128 / 80 \mathrm{~mm} \mathrm{Hg}$. The patient was subsequently admitted to hospital and was discharged well following review of his regular medications.

\section{CASE 2}

An 84-year-old man was brought to the ED by ambulance. He had been feeling generally unwell for $24 \mathrm{~h}$. When the ambulance arrived the patient collapsed, with an initial heart rate of 40, falling to 20 and with a blood pressure of $70 / 55 \mathrm{~mm} \mathrm{Hg}$. Atropine $2.5 \mathrm{mg}$ and $500 \mathrm{ml} \mathrm{0.9 \%}$ saline were administered with no response. $\mathrm{He}$ had type 2 diabetes with a history of hypertension. Medications were aspirin, rosiglitazone, lisinopril, digoxin, gliclazide, bendroflumethiazide and diltiazem.

On arrival in the $\mathrm{ED}$ his pulse rate remained 20 , but with a blood pressure of $103 / 79 \mathrm{~mm} \mathrm{Hg}$. The ECG revealed a bizarre wide complex rhythm with no identifiable $\mathrm{P}$ waves (fig 2).

An arterial blood gas revealed a $\mathrm{pH}$ of 7.16 and potassium of $8.02 \mathrm{mmol} / \mathrm{l}$, with hyperkalaemia confirmed by a subsequent laboratory report of $8.3 \mathrm{mmol} / \mathrm{l}$. Calcium chloride $2 \mathrm{~g}$ was administered intravenously over 5 minutes, with immediate resolution of the bradycardia and the patient became fully alert. The heart rate improved to 84 with a blood pressure of 110/84 mm Hg. Further treatment for hyperkalaemia was administered and the patient subsequently made a full recovery.

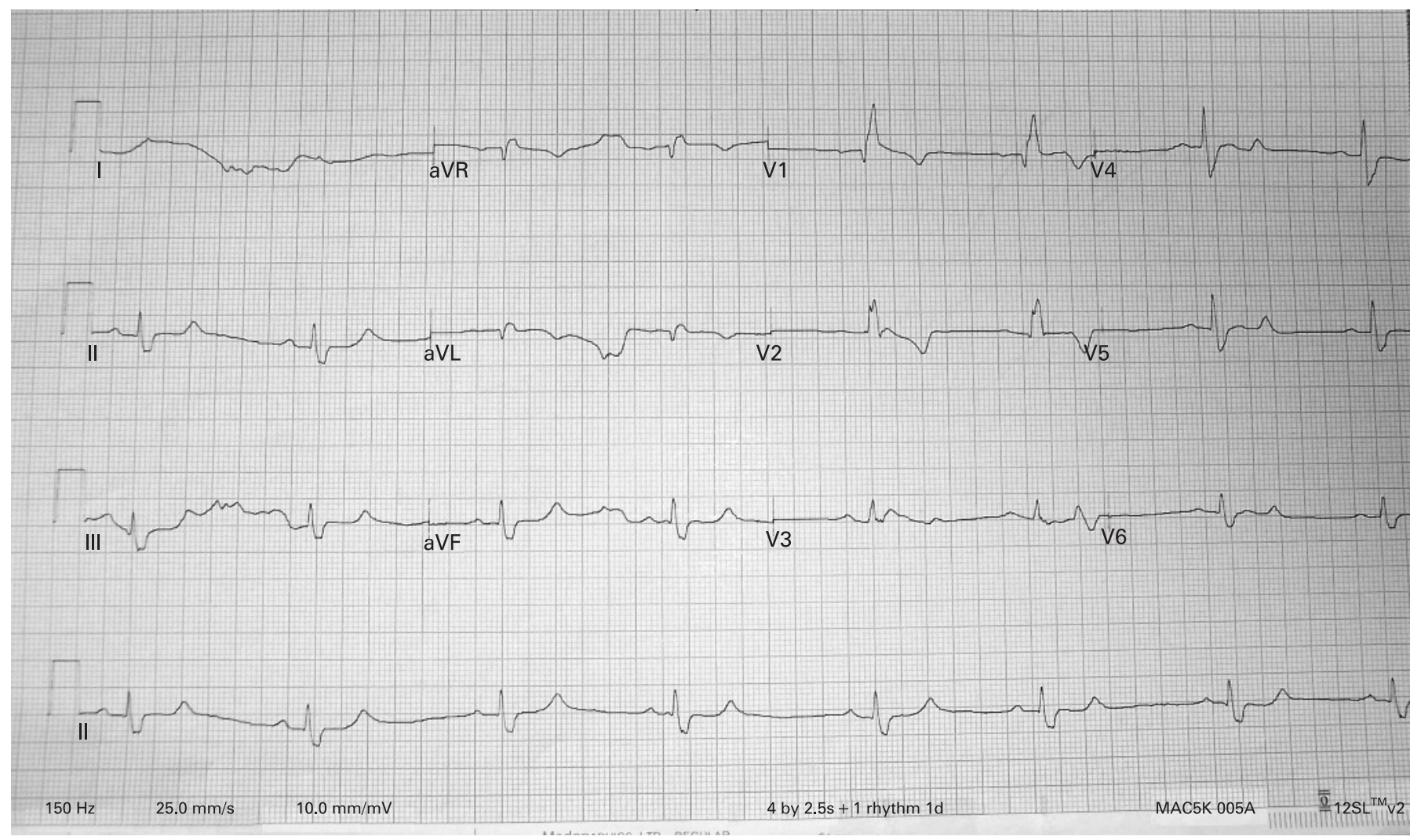

Figure 1 Case 1: Initial electrocardiogram showing sinus bradycardia. 


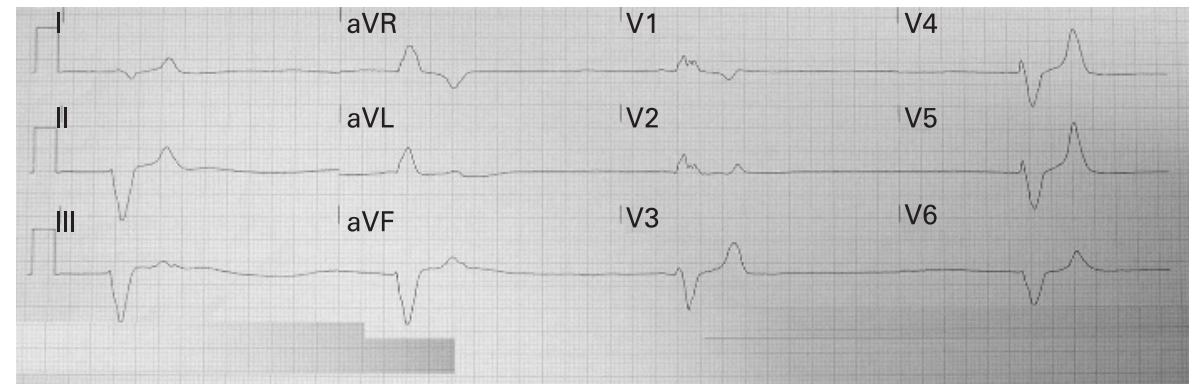

Figure 2 Case 2: Initial electrocardiogram showing wide ORS complexes without discernable P waves.

\section{DISCUSSION}

The classic ECG signs of hyperkalaemia include: ${ }^{1}$

- Narrow-based peaked T waves (serum potassium $>5.5 \mathrm{mmol} / \mathrm{l}$ )

- Prolonged ORS and PR interval and flattened P waves $(>6.5 \mathrm{mmol} / \mathrm{l})$

- Intraventricular blocks, bundle branch blocks (>8.0 mmol/l)

- Eventual "sine-wave" ORS complexes, ventricular fibrillation, asystole

Many other ECG abnormalities have, however, been attributed to hyperkalaemia. These include both tachycardia and bradycardia and also idioventricular rhythm, heart block and a pseudo-infarction pattern with massive ST segment elevation. ${ }^{1}{ }^{2}$

Life-threatening hyperkalaemia causing profound bradycardia can appear to resemble complete heart block (as in case
2); however, there is an absence of $\mathrm{P}$ waves. A UK case report has previously highlighted the resolution of hyperkalaemic bradycardia with intravenous calcium administration. ${ }^{3}$ This phenomenon is not described in the emergency medicine literature, however, and appears to be poorly recognised as a cause of symptomatic bradycardia.

The effects of calcium occur within 1 3 minutes but last for only 30-60 minutes ${ }^{2}$ and definitive treatment is needed to lower serum potassium levels. When arrhythmias are present, a wealth of anecdotal and animal data suggest that intravenous calcium is effective in treating arrhythmia. Further studies of the optimal use of combination treatments and of the adverse effects of treatments are needed. ${ }^{4}$

The patient in case 2 took digoxin regularly. Because hypercalcaemia can potentiate digitalis toxicity, calcium should be used in patients taking digitalis only if there is a loss of $P$ waves or a widened ORS complex. In this situation, calcium gluconate should be diluted in $100 \mathrm{ml} \mathrm{5 \%}$ dextrose and infused over 30 minutes. ${ }^{2}$

Bradycardia secondary to hyperkalaemia is not a commonly recognised ECG finding. These cases show the value of both rapid bedside testing of serum potassium in the ED (by arterial blood gas analyser) and clinicians having an awareness of the many and varied ECG presentations of hyperkalaemia.

${ }^{1}$ Emergency Department, Bristol Royal Infirmary, Bristol, UK; ${ }^{2}$ Great Western Hospital, Swindon, UK

Correspondence to: Dr T J Slade, Bristol Royal Infirmary, Emergency Department, Marlborough Street, Bristol BS2 8HW, UK; tobyslade@hotmail.com

Accepted: 28 February 2008

Competing interests: None declared.

\section{Patient consent: Obtained.}

Emerg Med J 2008;25:611-612.

doi:10.1136/emj.2007.054114

\section{REFERENCES}

1. Mattu A, Brady WJ, Robinson DA. Electrocardiographic manifestations of hyperkalaemia. Am J Emerg Med 2000;18:721-9.

2. Parham WA, Mehdirad AA, Biermann KM, et al. Hyperkalaemia revisited. Tex Heart Inst J 2006;33:40-7.

3. Noble K, Isles C. Hyperkalaemia causing profound bradycardia. Heart 2006;92:1063.

4. Mahoney BA, Smith WA, Lo DS, et al. Emergency interventions for hyperkalaemia. Cochrane Database Syst Rev 2005 Apr;18(2):CD003235. 